\title{
Performance Analysis of Selective Opportunistic Spectrum Access With Traffic Prediction
}

\author{
Guangxiang Yuan, Ryan C. Grammenos, Yang Yang, Member, IEEE, and Wenbo Wang, Member, IEEE
}

\begin{abstract}
In cognitive radio (CR) networks, the ability to capture a frequency slot for transmission in an idle channel has a significant impact on the spectrum efficiency and quality of service $(\mathrm{QoS})$ of a secondary user (SU). The radio frequency (RF) frontends of an SU have limited bandwidth for spectrum sensing with the target frequency bands dispersed in a discontinuous manner. This results in the SU having to sense multiple target frequency bands in a short period of time before selecting an appropriate idle channel for transmission. This paper addresses this technical challenge by proposing a selective opportunistic spectrum access (SOSA) scheme. With the aid of statistical data and traffic prediction techniques, our SOSA scheme can estimate the probability of a channel appearing idle based on the statistics and choose the best spectrum-sensing order to maximize spectrum efficiency and maintain an SU's connection. By means of doing so, this SOSA scheme can preserve the QoS of an SU while improving the system efficiency. In contrast to previous work, we consider the practical issues encountered by an $\mathrm{SU}$ in a wireless environment, such as discontinuous target frequency bands and limited spectrumsensing ability. We examine the spectrum-sensing scheme in terms of packet loss ratio (PLR) and throughput. The simulation results show that the proposed SOSA scheme can decrease the probability of packet losses in the discontinuous spectrum environment and improve the spectrum efficiency.
\end{abstract}

Index Terms-Cognitive radio (CR), spectrum sensing, traffic prediction.

\section{INTRODUCTION}

C URRENT radio systems employ inflexible spectrum allocation strategies, resulting in inefficient spectrum utilization [1]. Cognitive radio (CR), which has attracted the attention of many researchers across the globe, is a novel technology aimed at more efficiently utilizing the spectrum [2].

Manuscript received May 25, 2009; revised October 2, 2009. First published December 28, 2009; current version published May 14, 2010. This work was supported in part by the China Scholarship Council under the postgraduate student exchange program by the Ministry of Science and Technology of China under Grant 2009DFB13080, by the National Basic Research Program of China (973) under Grant 2007CB310602, and by the Research Councils U.K. under the U.K.-China Science Bridges Project: R\&D on (B)4G Wireless Mobile Communications (EP/G042713/1). The review of this paper was coordinated by Prof. H. Aghvami.

G. Yuan and W. Wang are with the Wireless Signal Processing and Networks Laboratory, Beijing University of Posts and Telecommunications, Beijing 100876, China (e-mail: gxyuan@gmail.com; wbwang@bupt.edu.cn).

R. C. Grammenos is with the Department of Electronic and Electrical Engineering, University College London, WC1E 7JE London, U.K. (e-mail: r.grammenos@ee.ucl.ac.uk).

Y. Yang is with the Department of Electronic and Electrical Engineering, University College London, WC1E 7JE London, U.K., and also with the Shanghai Research Center for Wireless Communications, Chinese Academy of Sciences, Shanghai 200335, China (e-mail: y.yang@ee.ucl.ac.uk).

Color versions of one or more of the figures in this paper are available online at http://ieeexplore.ieee.org.

Digital Object Identifier 10.1109/TVT.2009.2039155
CR technology enables a secondary user (SU) with cognitive access ability to use the idle channel resources of the primary radio systems. This idle channel resource, which is temporarily unoccupied by the primary user (PU), can be used by the SU to exchange information, provided it does not interfere with the smooth communication of the primary system [3], [4].

Current research in CR focuses on solving two main challenges, namely, 1) the avoidance of interference to the normal communication of primary systems and 2) the performance optimization of SU's transmission. For the former challenge, several methods for accurate signal detection have been proposed, including matched filter detection, energy detection, and cyclostationary feature detection [3]. To combat the negative effect caused by fading to the transmitter detection accuracy, a novel detection method based on CR user cooperation is proposed in [5] and [6]. As far as the SU's performance is concerned, SUs within the CR system should intelligently search and exploit vacant channel resources via dynamic spectrum access. Nevertheless, SUs do not have the ability to gather data from the primary systems; hence, they can only obtain information regarding the channel status of different frequency bands using periodic spectrum detection, which in turn consumes a nonnegligible length of time [11].

With regard to the deployment of wireless access networks in practice, SUs will be present in an environment comprising numerous wireless access networks spanning a wide spectrum range in a discontinuous manner [1]. Consequently, the $\mathrm{SU}$ is likely to lose some transmission opportunities made available from vacant channels, unless it can support spectrum sensing over a large frequency range spanning several gigahertz. Stateof-the-art radio frequency hardware capable of sensing weak signals over a large dynamic range, such as multispeed analogto-digital converters with a high resolution, wideband antennas, and highly linear power amplifiers, are still subject to many application constraints [3]. Thereby, an SU will have limited ability to carry out spectrum sensing and access, leading to an overall decrease in throughput.

In this paper, we propose a generic selective opportunistic spectrum access (SOSA) framework to carry out intelligent and selective spectrum sensing and access. This SOSA framework enables an SU to sense and select target spectrum bands in an optimum order while achieving quaity-of-service (QoS) requirements and maximizing spectrum efficiency. This is achieved with the aid of traffic prediction, which allows an SU to determine the optimum channel-sensing order by taking into account the probability of a channel appearing idle in the next time slot and its QoS requirements. In this paper, we also define the criteria and devise a theoretical model for evaluating 
system performance under the application of prediction-based spectrum sensing and access. We derive the theoretical upper bound for an SU's system performance by employing traffic prediction, which in turn could serve as a performance evaluation criterion for different traffic prediction methods in CR networks.

The rest of this paper is organized as follows: In Section II, the related work on traffic prediction and its application in CR are summarized. In Section III, the proposed generic intelligent spectrum sensing cycle is described, and the system parameters for our research scenario, the PU traffic model, and the SOSA scheme are defined. Section IV examines the proposed selective spectrum sensing and access strategy in detail. In Section V, the performance evaluation models are presented, and the SOSA scheme performance is investigated. The simulation results are analyzed in Section VI. Finally, the conclusions are given in Section VII.

\section{RELATED WORK}

The ability to predict the variation of parameters reflecting network conditions, such as bandwidth, interference level, bit rate, and bit error rate, in future time intervals is a key challenge in network management and control. As a result, traffic prediction is becoming increasingly important. Previous work has focused on improving network efficiency and QoS performance through traffic control and avoidance of traffic congestion. Fuzzy logic and linear autoregressive prediction algorithms have been implemented for this case [14], [15].

CR technology enables users to share the available spectrum in an opportunistic manner. Current research in CR networks focuses on optimizing specific parameters, such as link outage probability and delay, rather than capturing transmission opportunities at the physical layer to maximize the overall system throughput. As far as spectrum handoff is concerned, [8] and [9] analyze the probability of link maintenance during a spectrum handoff by employing different spectrum handoff schemes or a different number of redundant channels, respectively. In [10], a protocol is proposed to support link maintenance during a spectrum handoff for orthogonal frequency-division-multiplexingbased CR systems. In recent years, several traffic-prediction methods have been introduced into CR technology. Reference [13] presents different prediction rules for different types of ON/OFF traffic models with the aim of minimizing the number of handovers between channels, as well as the associated delay. In [14], an autoregressive spectrum hole prediction model is presented, which adopts an AR-2 model with a Kalman filter to reduce the collisions between PUs and SUs. In [15], a proactive spectrum-access approach is introduced, which differs from the existing reactive sense-and-avoid approaches in that channel histories are utilized to make predictions regarding future spectrum conditions. Furthermore, the idea of predictive dynamic spectrum access is also explored and developed in [15], which is then implemented in [16] using cyclostationary detection and hidden Markov models.

Although several models that employ traffic prediction have been proposed, none of them consider traffic prediction and spectrum sensing in conjunction in a practical heterogeneous

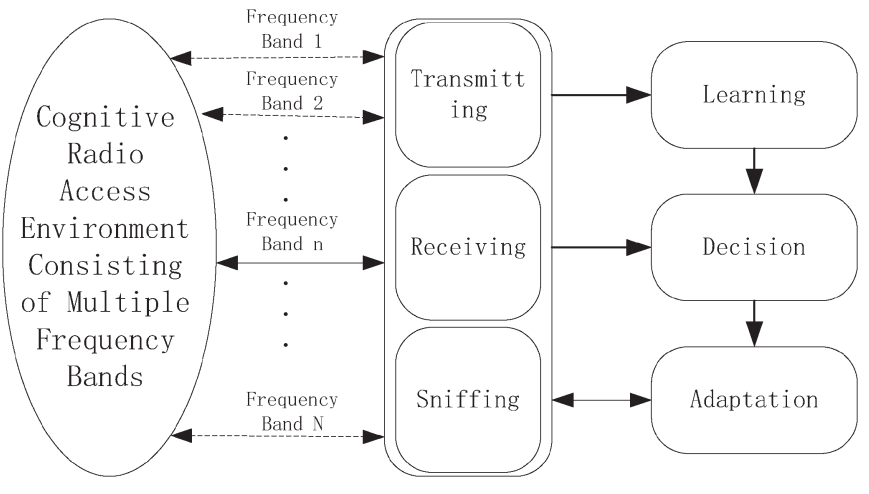

Fig. 1. Generic selective spectrum-sensing and access cycle.

wireless network environment where SUs have limited sensing and access ability. Furthermore, there is still no unified theoretical model for evaluating system performance when proactive spectrum selection and sensing are employed.

\section{System Model and Problem Formulation}

\section{A. Generic Selective Spectrum-Sensing Cycle}

In [4], the SU is defined as a machine that can learn from the surrounding environment in an intelligent manner and adjust its transmission parameters to meet certain objectives, such as link reliability and transmission rate, in the light of the learning outcome. We can divide the $\mathrm{CR}$ entire sensing and access process into four parts: 1) sniffing; 2) learning; 3) decision; and 4) adaption.

1) Sniffing stage-During this stage, relevant information concerning the surrounding wireless access networks is collected and stored, such as bandwidth, traffic operation, interval duration, and channel quality.

2) Learning stage-This phase involves the analysis of statistical data, the modeling of PU traffic, and the estimation of parameters serving as inputs to the spectrum sensing and access strategy.

3) Decision stage - In this stage, the spectrum sensing order is determined, as well as the access scheme for the next time unit, depending on the values of the input parameters obtained from the learning stage.

4) Adaption stage-This final phase involves adjusting the transmitter and receiver parameters according to the outputs acquired from the spectrum-sensing and access strategy.

The generic spectrum-sensing and access framework, which is based on the foregoing four operations, is illustrated in Fig. 1. First, using a sniffing process, the SU collects, stores, and updates information regarding the usage of target frequency bands. During the learning stage, the SU will estimate the PU traffic pattern and the relevant traffic parameters with the aim of predicting the PU traffic trend for a future time interval. The $d e$ cision stage is where the SU will determine the optimum sensing order based on QoS requirements, the probability of each channel appearing idle in the next time interval, and the transmission capability of each frequency band under consideration. The spectrum sensing and access operation will take place 
during the adaptation phase based on the outcome obtained from the decision phase. This adaptation may involve tuning transceiver parameters to adapt to the instantaneous channel variations. In our work, we assume that spectrum overlay is the only access method available to an SU, unless stated otherwise. Moreover, taking into account that, with spectrum overlay, the transceiver can be configured in a more straightforward fashion compared with the underlay method, we will also assume that the SU's transceiver transmits data with a fixed power, which will not cause interference to the PUs operation in the licensed spectrum.

\section{B. System Architecture and Notation}

We consider a distributed slotted wireless access environment where the multiple frequency bands span a wide spectrum range. In our work, these frequency bands are defined as primary channels and are independent of each other. All primary channels serve as target-sensing channels for an SU, with some of them acting as potential access channels for an SU in a specific time slot. The following notations are used throughout the remaining sections of this paper:

$N \quad$ total number of target frequency bands or primary channels that can be accessed by the SU;

$K$ random variable representing the number of channels that are idle in a time slot, where $0 \leq K \leq N$;

$i \quad$ time slot index in the primary system;

$w_{n}^{i} \quad$ channel state of the $n$th channel at time slot $i . \mathcal{W}_{n}(i)=1$ means that the channel is occupied by a PU, namely ON (Busy) state; $\mathcal{W}_{n}(i)=0$ means that the channel is not being used by a PU, namely OFF (Idle) state;

$T_{s} \quad$ time slot length in the primary system;

$C_{n}^{i} \quad$ transmission capacity of the target $\mathrm{SU}$ on the $n$th primary channel in the $i$ th time slot;

$t_{s} \quad$ average sensing time per primary channel;

$t_{h} \quad$ average handoff time between two primary channels;

$M \quad$ random variable representing the number of channels that are assumed to be busy in a time slot but are in fact idle, where $0 \leq M \leq K$;

$L \quad$ random variable representing the number of channels that are assumed to be idle in a time slot but are in fact busy, where $0 \leq L \leq N-K$;

$R \quad$ target transmission rate of an $\mathrm{SU}$;

$\alpha_{n} \quad$ mean value of the OFF-period for the $n$th primary channel;

$\beta_{n} \quad$ mean value of the ON-period for the $n$th primary channel;

$S \quad$ spectrum sensing time threshold;

$P_{e} \quad$ average probability of error associated with the predictor of an SU;

$P_{\text {off }}^{n, i} \quad$ predicted probability of the $n$th primary channel appearing idle in the $i$ th time slot;

$P_{\mathrm{on}}^{n, i}$ predicted probability of the $n$th primary channel appearing busy in the $i$ th time slot.

The following two sections present the design assumptions and the implementation issues associated with the PU and SU systems, respectively.

\section{PU Traffic Model}

PU traffic can generally be modeled in one of two distinct ways: using either a deterministic model or a stochastic model, depending on the traffic pattern of the primary channels under consideration. In the current $\mathrm{CR}$ research, the traffic activity for PUs operating on a licensed frequency band is modeled as an alternating renewal process consisting of busy and idle periods [3], [11], which correspond to the stochastic model. An example of this alternating renewal process is illustrated in Fig. 2(a). We use binary digits 0 and 1 to accordingly denote the idle state $(\mathrm{OFF})$ and the busy state $(\mathrm{ON})$ for each primary channel in every time slot. In the aforementioned literature, the $\mathrm{ON}$ and OFF periods of the primary channels are independent identically distributed (i.i.d.), where the alternating renewal process is modeled as a two-state birth-death process with death rate $\alpha_{n}$ and birth rate $\beta_{n}$. The lengths of the OFF and ON periods follow an exponential distribution with mean value equal to $\alpha_{n}$ and $\beta_{n}$, respectively [11].

\section{SU Spectrum Sensing and Access}

The spectrum-sensing procedure is indispensable to an $\mathrm{SU}$ since it entails two important functions that allow an SU to obtain access to primary systems. The first function validates the real state of the primary channels to be accessed to avoid interference. The second function involves collecting long-term usage data with regard to the activity taking place on the primary channels to aid traffic prediction. Existing spectrum sensing technologies cannot achieve faultless spectrum detection, and undetected errors degrade system performance [12]. However, physical layer issues are beyond the scope of this paper and, hence, are not dealt with in our work. Instead, we assume that the sensing results generated by the spectrum sensor of an $\mathrm{SU}$ are accurate enough to establish the state of a primary channel.

In each time slot, the SU senses all the primary channels one by one and transmits its data on the first idle channel that becomes available. Following transmission, the $\mathrm{SU}$ continues to sense the remaining primary channels to collect their channel state, which will subsequently be used to predict the channel states in the next time slot. The detailed channel-sensing and access process is illustrated in Fig. 2(b). It is evident that the overall time required to sense $N$ primary channels should not exceed the length of a time slot $T_{s}$; otherwise, the SU will not be able to learn the states of all primary channels, making it impossible to predict the channel state for the next time interval.

\section{Selective Opportunistic Spectrum Access}

In this section, we formulate our SOSA framework by presenting our theoretical models evaluating SU system performance under constant and variable bit rate (CBR and VBR) traffic.

The wireless access environment under consideration consists of $N$ primary channels, which are indexed as $1,2, \ldots, N$. These $N$ channels form a primary channel set $\Phi$, which conforms to no specific sequence criteria. The transmitter can access any of these channels without having to inform the 


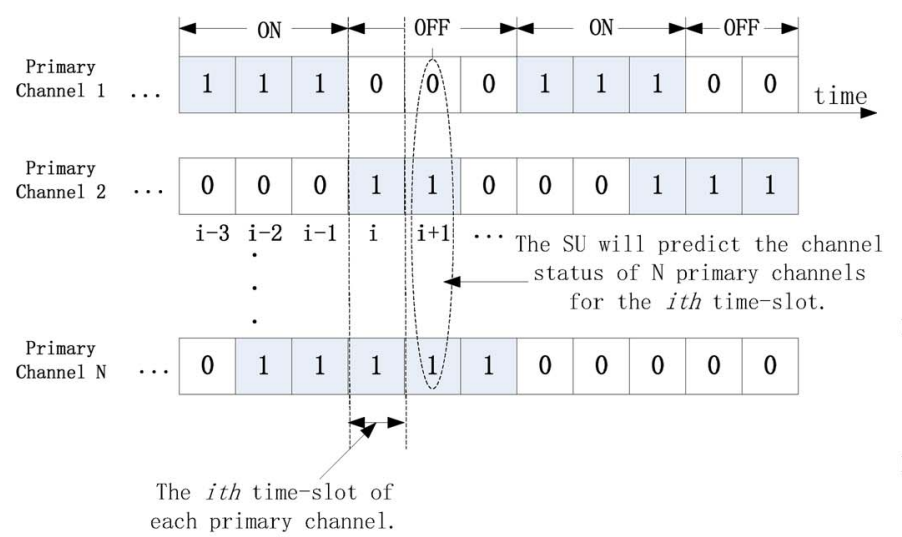

(a)

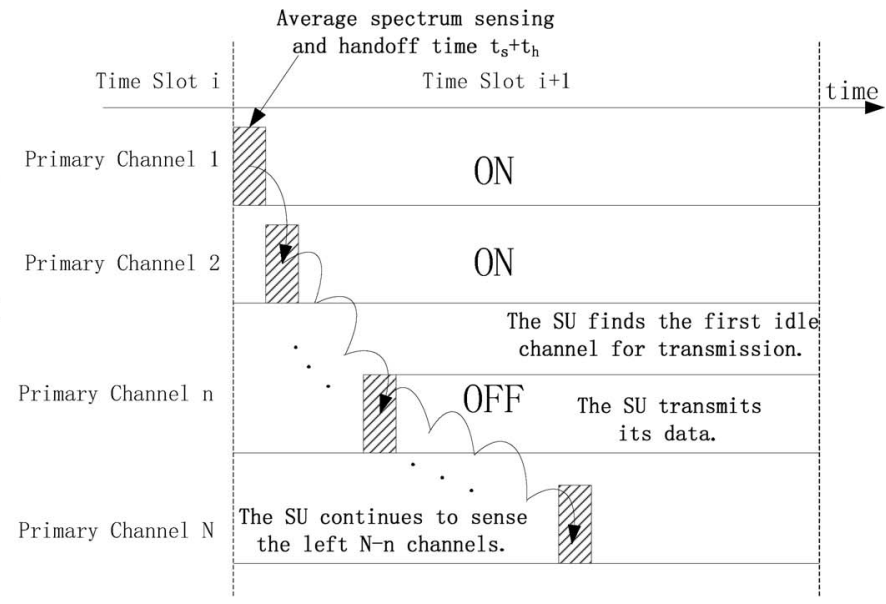

(b)

Fig. 2. Example of (a) the renewal process for a primary system and (b) the SU sensing process in a time slot.

primary system beforehand. Operations are carried out in the discrete time domain, which is indexed with $i$. At the start of each time slot, the SU needs to determine the sensing order by jointly considering the transmission capacity of each channel and its probability of appearing idle in the next time slot. These operations will take place during the learning and decision stages.

The target SU has a transmission capacity $C_{n}^{i}$ on the $n$th primary channel in time slot $i$. We have defined the variable $w_{n}^{i}$ to indicate the state of the $n$th primary channel in time slot $i$ taking binary values 0 or 1 . In this case, 0 represents an idle primary channel, whereas 1 represents a busy primary channel. If the SU finds an idle channel in time slot $i$, then the number of information bits in this time slot is $I(i)$. This is the theoretical product of an SU's channel capacity with its effective transmission time in one time slot having subtracted the time consumed for spectrum sensing and handoff. To protect a PU's signal, the SU is not permitted to transmit on a primary channel before sensing and detecting its real state.

We assume that the SU obtains the accurate channel state information vector $\mathbf{w}^{i}$ of $N$ primary channels, where $\mathbf{w}^{i}=$ $\left[w_{1}^{i}, w_{2}^{i}, \ldots, w_{N}^{i}\right]$, and $\mathbf{w}^{i} \in \mathcal{W}$, where $\mathcal{W}$ is the set of all possible primary channel state combinations. We also assume that the SU knows its transmission rate on the primary channels; in other words, the SU knows the channel capacity vector $\mathbf{C}^{i}$ in time slot $i$. This information can be collected via the sniffing process that takes place in each time slot. During the learning stage, the SU will predict the primary channel states for time slot $i+1$ based on the most recent channel state vector $\mathbf{w}^{i}$ and will update the channel history record accordingly. The SU can adjust the relevant parameters of its predictor to improve the accuracy of the results obtained; however, this depends on the initial design requirements outlined for the predictor. The prediction result can be represented by the vector $\hat{\mathbf{w}}^{i+1}$, which takes into account the probability of each primary channel being idle $P_{\text {off }}^{n, i+1}$ or occupied $P_{\text {on }}^{n, i+1}$ in time slot $i+1$. Without loss of generality, we can arrange the index of the $N$ primary channels according to their probability of being idle $P_{\text {off }}^{n, i+1}$ in time slot $i+1$ such that $P_{\text {off }}^{1, i+1} \geq P_{\text {off }}^{2, i+1} \geq \cdots \geq P_{\text {off }}^{N, i+1} \geq$ 0 . In contrast to previous work, the SU in our case does not predict the length of time for which a channel will remain idle. Taking such a criterion into account for the transmission of an SU's data would lead to a higher probability of outage compared with a slotted system scenario where the time unit is considered to be fixed. We formulate this process as

$$
\mathcal{L}: \mathcal{F}\left(\mathbf{w}^{i}, \boldsymbol{\alpha}, \boldsymbol{\beta}\right) \stackrel{R_{N}}{\longrightarrow} \hat{\mathbf{w}}^{i+1}, \quad n \in \Phi, \mathbf{w}^{i} \in \mathcal{W}
$$

where $\mathcal{L}$ indicates the learning process, $\mathcal{F}$ represents the prediction method used in the learning process, and $R_{N}$ is the traffic record of all $N$ primary channels. The data format for this record will be designed according to the prediction method employed. $\boldsymbol{\alpha}$ and $\boldsymbol{\beta}$ are the vectors for the death and birth rates, respectively, of the periods for all primary channels. The SU has to arrange the spectrum-sensing sequence of decreasing probability of a channel appearing idle while considering transmission requirements and sensing efficiency at the same time. This procedure can be modeled as follows:

$$
\mathcal{D}: \mathcal{G}\left(\hat{\mathbf{w}}^{i+1}, \mathbf{C}^{i+1}, \boldsymbol{\psi}\right) \longrightarrow \boldsymbol{U}^{*}, \quad n \in \Phi
$$

where $\psi$ is the set of transmission requirements for the $\mathrm{SU}, \mathcal{G}$ is the ordering strategy used by the SU to determine the sensing sequence for the primary channels, which jointly considers the transmission requirements of the SU and the sensing efficiency, and $\boldsymbol{U}^{*}$ is the optimum spectrum-sensing order acquired during the decision phase. The following pane summarizes the steps of our proposed spectrum sensing cycle:

\section{SOSA Scheme}

1) The $S U$ retrieves the channel statistics regarding the state of each obtained through sniffing and based on the predictor requirements defined at the start of time slot $i+1$.

2) The SU predicts the probability of each channel appearing idle or occupied in time slot $i+1$ using the predefined prediction model.

3) The SU uses the predetermined ordering strategy to arrange the sensing sequence for time slot $i+1$.

4) The SU begins to sense primary channels according to the sensing order generated in step 3). 
5) The SU locks on to the first idle channel that becomes available, provided that there are no special transmission requirements.

6) The SU continues to sense the remaining primary channels, finally recording the states of all $N$ primary channels in time slot $i+1$.

7) If deemed necessary, then the SU adjusts the predictor parameters according to the outcome obtained from the sniffing stage in the last time slot.

8) The SU repeats these steps until it reaches the last time slot.

For the purpose of performance evaluation and comparison, we adopt a relatively simple but effective channel-ranking method. This method takes the long-term statistical traffic characteristics as the critical input parameters to the learning process formulated in (1). The two input parameters $\boldsymbol{\alpha}$ and $\boldsymbol{\beta}$ are the vectors corresponding to the mean values of the OFF and ON periods of all primary channels, respectively. These mean values are obtained using the maximum-likelihood estimation method, which in turn uses statistical data relevant to the channel state of all primary channels. For simplicity, during the learning process, the channel state vector $\mathbf{w}^{i}$ is not considered in the channel-state-prediction process. The vector $\hat{\mathbf{w}}^{i+1}$, which represents the probability of a channel being idle in the next time slot, is determined by the channel's long-term statistical characteristics. If we denote $p_{n}$ as the probability of the $n$th primary channel appearing idle in the next time slot, then $p_{n}$ is given by

$$
p_{n}=\frac{\alpha_{n}}{\alpha_{n}+\beta_{n}}
$$

where $1 \leq n \leq N$. As channel-state statistical data are gradually collected over the period of time, the mean lengths of the $\mathrm{ON}$ and OFF periods, as well as the probability of each primary channel appearing idle in the next time slot, will be updated accordingly.

Equation (2) indicates that the channel capacity and the transmission requirements of the SU will be taken into account during the decision procedure. In our work, we assume that all primary channels have the same transmission capacities $C_{0}$ and that their channel-state variation is slow enough so that it does not distort the results obtained from our theoretical evaluation model. Hence, $\mathbf{C}^{i+1}$ in (2) is simplified into a constant vector. The input parameter $\psi$ in (2) reflects the SU's transmission requirements and should be considered in conjunction with the transmission capacity. The optimum channel-sensing sequence indicated by $\boldsymbol{U}^{*}$ should be arranged in order of decreasing probability according to the corresponding magnitudes defined in vector $\hat{\mathbf{w}}^{i+1}$.

\section{PERformance AnAlysis}

In the previous sections, we looked at the challenges encountered by an SU when wishing to capture a transmission opportunity. In this section, we will evaluate system performance in terms of two key performance metrics, namely, packet loss ratio (PLR) and throughput. In this paper, the transmission quality of an SU is evaluated under CBR and VBR traffic in terms of PLR and throughput, respectively. In the following two sections, we derive the theoretical models to evaluate the performance of $\mathrm{CR}$ systems with and without traffic prediction. We determine the system performance upper bound under the assumption that the SU has limited spectrum-sensing and access capability and is operating in a wireless environment, which consists of discontinuous target frequency bands. This performance upper bound could in turn be used to compare different traffic prediction and spectrum-sensing methods.

In Section III, we defined the random variable $K$ as the number of idle primary channels in one time slot $i$. Hence, the number of occupied primary channels in one time slot is $N-K$. Since all the primary channels are i.i.d., it follows that each channel has the same probability of being idle, which is denoted by $P$. Therefore, the random variable $k_{i}$ follows a binomial distribution with a mean value equal to $N P$.

\section{A. PLR}

For CBR traffic, the target SU needs to transmit $R$ information bits in one time slot. We define the packet loss as the event during which an SU cannot find an idle channel for transmission that has already sensed $S$ primary channels, where $S$ is the sensing threshold. In other words, $S$ is defined as the maximum number of channels the SU is permitted to sense to find a vacant channel. This threshold is determined by the transmission capacity of the target primary channel to be accessed by the $\mathrm{SU}$, as well as the number of information bits that need to be transmitted in one time slot [19]. $S$ is given by

$$
S=\left\lfloor\frac{T_{s}-R / C_{0}}{t_{s}+t_{h}}\right\rfloor
$$

where $1 \leq S<N$.

1) Spectrum Sensing Without Traffic Prediction: Without the aid of traffic prediction, capturing a transmission opportunity is totally dependent on the traffic activity taking place on each primary channel. Since the SU needs to transmit its data at a CBR, packet loss will occur when the SU cannot locate an idle primary channel for transmission within the sensing time threshold $S$. We assume that there are $K$ idle primary channels in time slot $i$. The probability that the SU cannot find an idle channel from $K$ idle primary channels is given by

$$
P_{\mathrm{wo}}^{i, K}=\prod_{j=1}^{S}\left(1-\frac{K}{N-j+1}\right), \quad 0 \leq K \leq N .
$$

This is the instantaneous packet loss probability for time slot $i$. As earlier defined, $K$ is a random variable following a binomial distribution with mean value $N P$ and represents the number of idle primary channels in time slot $i$. Hence, the average probability of packet loss for an SU in time slot $i$ is given by

$$
\begin{aligned}
P_{\mathrm{wo}}^{i} & =\sum_{k=0}^{N} P_{r}\left\{K_{i}=k\right\} \prod_{j=1}^{S}\left(1-\frac{K_{i}}{N-j+1}\right) \\
& =\sum_{k=0}^{N}\left(\begin{array}{c}
N \\
k
\end{array}\right) P^{k}(1-P)^{N-k} \prod_{j=1}^{S}\left(1-\frac{k}{N-j+1}\right) .
\end{aligned}
$$


TABLE I

Packet Loss Condition Analysis in Spectrum Sensing With TRAFFIC PREDICTION

\begin{tabular}{|c|c|c|c|}
\hline \multicolumn{3}{|c|}{ Parameters: K,L,M,S,N } & Type \\
\hline$K \leq N-S$ & $K>M$ & $K-M+L \leq S$ & $\mathrm{a}$ \\
\cline { 2 - 4 } & & $K-M+L>S$ & $\mathrm{~b}$ \\
\cline { 2 - 4 } & $K=M$ & $L \geq S$ & $\mathrm{c}$ \\
\cline { 2 - 4 } & & $L<S$ & $\mathrm{~b}$ \\
\hline$K>N-S$ & \multicolumn{2}{|c}{ N/A } & $\mathrm{a}$ \\
\hline
\end{tabular}

For the case where different primary channels have different traffic characteristics, the average PLR taking into account the sensing time threshold $S$ is given by

$$
P_{\text {wo }}^{\text {ave }}=\prod_{n=1}^{S}\left(1-p_{n}\right), \quad 1 \leq S \leq N .
$$

2) Spectrum Sensing With Traffic Prediction: In this scenario, the prediction result obtained for the next time slot is used to arrange the sensing order. In a similar fashion to the case without traffic prediction, the SU must find an idle primary channel within the sensing time threshold $S$ to prevent packet loss. Since a perfect predictor does not exist in practice, incorrect prediction results will give rise to errors that could lead to packet loss. We can identify three different scenarios in relation to packet loss.

1) No packet loss takes place.

2) Packet loss may occur.

3) Packet loss will definitely occur.

The three foregoing cases are summarized in Table I. From Table I, we can see that packet loss could occur as a result of the simultaneous interaction between multiple random variables. Packet loss may take place under two conditions, i.e., $(K \leq N-S, M<K, K-M+L>S)$ and $(K \leq N-$ $S, M=K, L<S)$, whereas it will definitely occur if the condition $(K \leq N-S, M=K, L \geq S)$ is met. The number of primary channels predicted to be idle in the next time slot is $K-M+L$. Their joint probability density function (pdf) has a tight relationship with the probability of packet loss taking place. We define the joint pdf of these three random variables as $V_{K, M, L}(k, m, l)$. For the purpose of deriving a unified formula for evaluating different prediction methods, we define $P_{e}$, with $P_{e}>0$, as the average probability that the SU will predict the state of a primary channel in the next time slot incorrectly. For a detailed derivation of $V_{K, M, L}(k, m, l)$, see the Appendix.

1) $K \leq N-S, M=K, L \geq S$ : According to the joint pdf $G_{K, M, L}(k, m, l)$, the probability for this case is given by

$$
P_{w, 1}^{i}=V_{K, M, L}(N-S, N-S, L \geq S) .
$$

2) $K \leq N-S, M=K, L<S$ : For this case, the difference between the number of primary channels $L$ (predicted incorrectly as being idle) and the sensing time threshold $S$ determines the probability of packet loss taking place and is given by

$$
P_{w, 2}^{i}=V_{K, M, L}(N-S, N-S, S) \prod_{j=1}^{S-L}\left(1-\frac{K}{N-L-j+1}\right)
$$

where $K$ follows a binomial distribution with mean value $(N-S) P$ and has a numerical range $[0, N-S]$.

3) $K \leq N-S, M<K, K-M+L>S$ : For this case, the difference between the number of primary channels predicted to be idle in the next time slot and the sensing time threshold $S$ determines the probability of packet loss taking place. If this difference is greater or equal to the number of primary channels that are idle in the next timeslot, which is denoted by $L \geq S$, then it is likely that packet loss will occur. In a similar fashion, the probability of incorrect prediction is determined by the number of channels that have been predicted as being idle in the next time slot when in actual fact they are busy. Therefore, the probability of packet loss in this third case is given by

$$
\begin{aligned}
P_{w, 3}^{i}=V_{K, M, L}(N-S, K, L \geq S) & \\
& \cdot \prod_{j=1}^{S}\left(1-\frac{K-M}{K-M+L-j+1}\right)
\end{aligned}
$$

where $K$ follows a binomial distribution with mean value $(N-S) P$ and has a numerical range $K$ is $[0, N-S]$. In summary, the PLR for an SU carrying out spectrum sensing with traffic prediction is given by

$$
P_{w}^{i}=\sum_{j=1}^{3} P_{w, j}^{i} .
$$

In this situation, where traffic prediction is used, we assume that the SU will always sense the channels in an optimum order. In other words, the primary channel to be sensed first will be the one with the highest statistical average probability of appearing idle in the next time slot with the remaining channels arranged in order of decreasing probability and renumbered accordingly from 1 to $N$, where the primary channel with index 1 is the channel with the highest statistical average probability of being idle and will be sensed first. Defining $P_{e}$ as the predictor's average probability of error, the average PLR for an $\mathrm{SU}$ employing traffic prediction is given by

$$
P_{w}^{\text {ave }}=\prod_{j=1}^{S}\left(1-p_{n}+P_{e} p_{n}\right) .
$$

3) Performance Upper Bound: In view of the fact the perfect prediction is not feasible in practice, it is obvious that the real state of all the primary channels at a future point in time cannot be determined. Nevertheless, if we assume that the SU has precognition ability, meaning it has perfect knowledge of the state of each primary channel in the next time slot, then no time will be spent to carry out spectrum sensing and handoff. Hence, for this case, packet loss will occur if and only if there are no idle channels available in the time slot under consideration. Based on the foregoing analysis, the PLR of an SU with precognition ability is given by

$$
P_{\text {pre }}=\prod_{n=1}^{N} \frac{\alpha_{n}}{\alpha_{n}+\beta_{n}}=\prod_{n=1}^{N}\left(1-p_{n}\right) .
$$


Since the $N$ primary channels are i.i.d., the packet loss probability is $(1-P)^{N}$.

\section{B. Throughput}

In Section III, we defined the channel-transmission capacity of an SU in one time slot. In this section, we analyze and model the throughput of an SU considering the same three scenarios identified in the previous section, which examined the PLR. Contrary to the PLR scenarios where the sensing threshold $S$ was the key parameter, in the following cases, we assess how fast an SU can locate the first idle primary channel. The corresponding time required for this operation, which is independent of $S$, directly influences the throughput performance. Hence, $K$ is still defined as the number of idle primary channels in time slot $i$; however, the number of channels the $\mathrm{SU}$ has to sense before arriving at the first idle channel is denoted by $\gamma_{i}^{K}$, where $\left(1 \leq \gamma_{i}^{K} \leq N\right)$. Consequently, the throughput of an SU in time slot $i$ is given by

$$
I(i)=C_{0}\left[T_{s}-\gamma_{i}^{K}\left(t_{s}+t_{h}\right)\right] .
$$

1) Spectrum Sensing Without Traffic Prediction: In this situation, where traffic prediction is not employed, the number of idle primary channels and their distribution in a time slot determine the throughput of an $\mathrm{SU}$ in this time slot. We assume that there are $K_{i}$ idle primary channels in time slot $i$. The probability of finding the first idle primary channel after sensing $j$ primary channels is

$P_{\text {wo }}^{i, K}=\prod_{m=1}^{j-1}\left(1-\frac{K_{i}}{N-m+1}\right) \frac{K_{i}}{N-j+1}, \quad 1 \leq j \leq N-K+1$.

Similar to the previous section, $K_{i}$ is a random variable following a binomial distribution with mean value $N P$. The average sensing time required to hit upon the first idle primary channel in time slot $i$, which is measured in terms of the number of channels sensed before the first idle channel is found, is given by

$$
\begin{aligned}
\bar{\gamma}_{\mathrm{wo}}^{i}= & \sum_{k=0}^{N} P_{r}\left\{K_{i}=k\right\} \cdot \sum_{j=1}^{N-K+1} j \cdot P_{\mathrm{wo}}^{i, K} \\
= & \sum_{k=0}^{N} \sum_{j=1}^{N-k+1}\left(\begin{array}{l}
N \\
k
\end{array}\right) P^{k}(1-P)^{N-k} \frac{j k}{N-j+1} \\
& \cdot \prod_{m=1}^{j-1}\left(1-\frac{k}{N-m+1}\right) .
\end{aligned}
$$

Therefore, we can substitute (16) into (14) and express the instantaneous throughput of an SU in time slot $i$ as

$$
\begin{aligned}
\bar{I}_{\mathrm{wo}}^{i}=C_{0}\left\{T_{s}-\right. & {\left[\sum_{k=0}^{N} \sum_{j=1}^{N-k+1}\left(\begin{array}{c}
N \\
k
\end{array}\right) P^{k}(1-P)^{N-k} \frac{j k}{N-j+1}\right.} \\
& \left.\left.\cdot \prod_{m=1}^{j-1}\left(1-\frac{k}{N-m+1}\right)\right]\left(t_{s}+t_{h}\right)\right\}
\end{aligned}
$$

Based on (17), the average throughput can be expressed as

$$
\begin{aligned}
\bar{I}_{\mathrm{wo}}=C_{0}\left[T_{s}-\right. & \left.\bar{\gamma}_{\mathrm{wo}}\left(t_{s}+t_{h}\right)\right] \\
& -\prod_{i=1}^{N}\left(1-p_{i}\right) \cdot C_{0}\left[T_{s}-N\left(t_{s}+t_{h}\right)\right] .
\end{aligned}
$$

where $\bar{\gamma}_{\text {wo }}$ represents the average sensing time and can be computed using the following formula:

$$
\bar{\gamma}_{\mathrm{wo}}=\sum_{i=0}^{N} i \prod_{j=1}^{i-1}\left(1-p_{j}\right) p_{i} .
$$

2) Spectrum Sensing With Traffic Prediction: Once again, when traffic prediction is employed, the SU determines the optimum spectrum sensing sequence allowing the primary channels that have a high probability of appearing idle in the next time slot to be given a higher priority for sensing. Each channel is associated with the probability of being idle $P_{\text {off }}^{n, i}$, as well as the probability of being busy $P_{\mathrm{on}}^{n, i}$, in the next time slot. To evaluate the instantaneous throughput of an $\mathrm{SU}$ in time slot $i$, we require the average spectrum sensing time $\bar{\gamma}_{w}$. The probability that an SU locates the first idle primary channel having sensed $j$ primary channels in one time slot with $K$ idle primary channels is given by

$$
P_{w}^{i, K}=\prod_{m=1}^{j-1} P_{\mathrm{on}}^{m, i} P_{\mathrm{off}}^{j, i}, \quad 1 \leq j \leq N-K+1 .
$$

Equation (20) indicates that $P_{w}^{i, K}$ depends on $P_{\text {on }}^{n, i}$ and $P_{\text {off }}^{n, i}$ whose accuracy varies according to the predictor's capability. Evidently, different prediction methods would yield different prediction results for the same statistical data. Taking $P_{e}$ to be the average probability of error as before, where $P_{e}>0,(20)$ can be rewritten as

$$
P_{w}^{i, K}=\left(1-P_{e}\right) P_{e}^{j-1}, \quad 1 \leq j \leq N-K+1
$$

with the average sensing time of an SU using traffic prediction given by

$$
\begin{aligned}
\bar{\gamma}_{w} & =\sum_{k=0}^{N} P_{r}\{K=k\} \cdot \sum_{j=1}^{N-k+1} j \cdot P_{w}^{i, k} \\
& =\sum_{k=0}^{N} \sum_{j=1}^{N-k+1}\left(\begin{array}{l}
N \\
k
\end{array}\right) P^{k}(1-P)^{N-k} j\left(1-P_{e}\right) P_{e}^{j-1} .
\end{aligned}
$$

Substituting (22) into (14), we obtain the average throughput of an $\mathrm{SU}$, which is expressed as

$$
\begin{gathered}
\bar{I}_{w}=C_{0}\left\{T_{s}-\left[\sum_{k=0}^{N} \sum_{j=1}^{N-k+1}\left(\begin{array}{l}
N \\
k
\end{array}\right) P^{k}(1-P)^{N-k} j\left(1-P_{e}\right)\right.\right. \\
\left.\left.\cdot P_{e}^{j-1}\right]\left(t_{s}+t_{h}\right)\right\} \cdot
\end{gathered}
$$

3) Performance Upper Bound: The throughput of an SU with precognition ability, implying the same definition as that 
given in the section for PLR, is expressed as

$$
I_{\text {pre }}=C_{0} T_{s}\left[1-\prod_{n=1}^{N}\left(1-p_{n}\right)\right] .
$$

Since all primary channels are i.i.d., the throughput of an SU with precognition ability can be written as $C_{0} T_{s}\left(1-P^{N}\right)$.

\section{Simulation Results And AnAlysis}

In the previous sections, we introduced and developed our proposed selective opportunistic spectrum-sensing and access cycle. In this section, we present the corresponding simulation results, which were generated using MATLAB, as well as the numerical results obtained from theory. All primary channels have the same traffic model but with different traffic characteristics. The lengths of the idle and busy channel state periods follow an exponential distribution. A datasheet was implemented to record the past and current primary channel states using a discrete time-domain series. In our simulation, we randomly generate the birth and death rates for each primary channel and generate sufficient traffic records to enable us to estimate the average probability of each primary channel being idle. The magnitudes of these probabilities are then used to determine the optimum spectrum-sensing order.

\section{A. PLR Analysis}

In this section, we compare the PLRs for the three scenarios identified earlier, namely, the spectrum-sensing cases with and without traffic prediction, as well as the system performance upper bound. Packet loss occurs when the SU fails to find an idle channel during the sensing time threshold $S$.

In Fig. 3, we compare the results under varying threshold values for a fixed number of primary channels. In Fig. 3, the curve with the lowest PLR represents the case where the SU has precognition ability, which is impossible to achieve in practice. Nevertheless, this curve could be used as the criterion for evaluating different prediction methods. We also find that this curve representing the system's performance upper bound tends to zero. This is due to the sporadic event that all $N$ primary channels are busy in that time slot. The curves corresponding to the case without traffic prediction almost overlap with each other, indicating that our simulation results are in accordance with those obtained from theory. This implies that the proposed theoretical model is highly accurate for evaluating the CR system performance for an SU operating in a discontinuous spectrum environment with limited spectrum-sensing and access ability.

The middle curve marked with circular points represents the PLR when the SU uses traffic prediction. In our simulation, we adopt a relatively simple prediction and channel-ordering method. The probability of a primary channel being idle is the sole parameter used to determine the sensing order. This curve corresponding to the case with traffic prediction should always lie between the curve representing the lowest PLR and the curve corresponding to the case where the sensing order remains unaltered. Employing more sophisticated prediction techniques would yield significant performance gains.

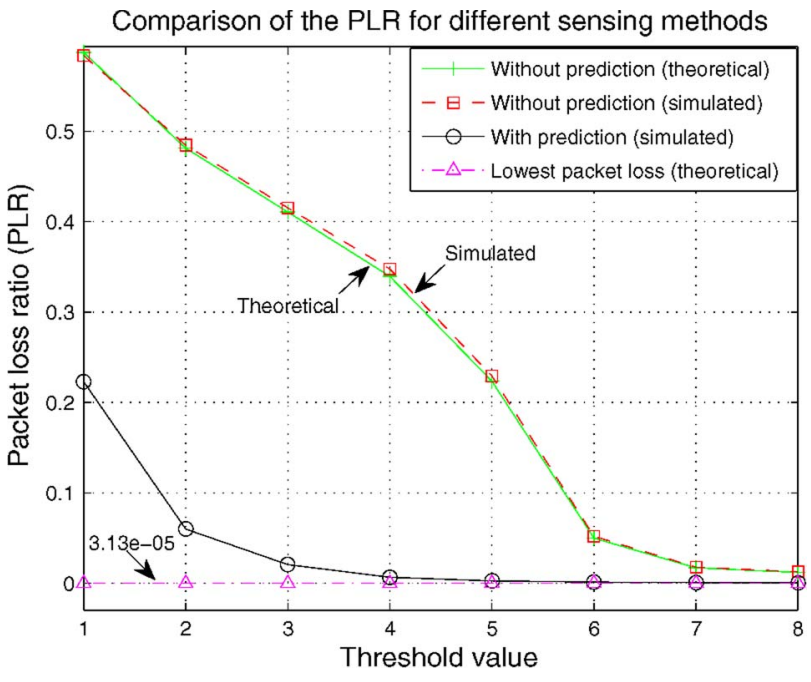

Fig. 3. PLRs for an increasing threshold value (under a fixed number of primary channels; $N=20$ ).

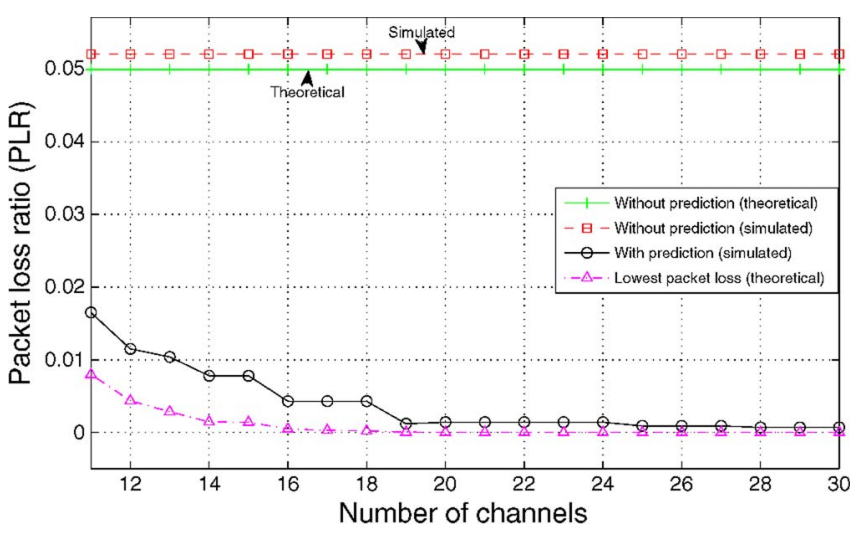

Fig. 4. PLRs for an increasing number of primary channels (under a fixed sensing time threshold value; $S=4$ ).

Finally, we see that the PLR decreases for an increasing threshold value. This is due to the fact that the probability of an idle channel being available increases as we increase the time given to an SU to sense the primary channels. On the contrary, the system performance upper bound remains constant at all times since it is independent of the sensing time threshold $S$.

Similar trends were obtained when the number of primary channels was increased from 10 to 30 under a fixed threshold value. The results are illustrated in Fig. 4. Once again, the two curves representing the simulation and theoretical results for the case where traffic prediction is not employed almost overlap with each other. The curve corresponding to the situation where the SU uses traffic prediction lies in between the other curves, in a similar manner to that in Fig. 3.

As can be seen in Fig. 4, the PLR for the case without traffic prediction does not improve as we increase the number of available primary channels. This is due to the fact that the sensing order in this scenario remains unaltered, meaning that the channels will not be arranged in such a way that would allow those with a higher probability of appearing idle to be sensed first during the spectrum sensing cycle. Hence, even if the total number of primary channels increases with more transmission opportunities becoming available in each time 


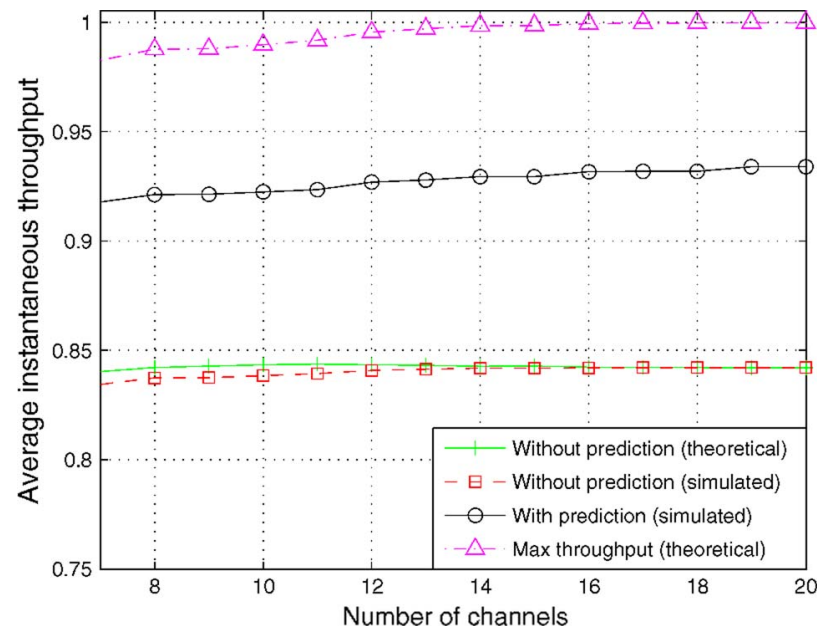

Fig. 5. Average throughput for an increasing number of primary channels (under a fixed sensing and handoff time; $t_{s}+t_{h}=0.05$ ).

slot, the transmission opportunities for the first $S$ primary channels will never change if the SU does not employ traffic prediction. This is clearly demonstrated in Fig. 4. On the other hand, the PLR for the other two cases will decrease as a result of finding transmission opportunities earlier.

\section{B. Throughput}

In this section, we evaluate the average throughput in each timeslot with the number of primary channels and the time consumed for spectrum sensing and handoff acting as the input parameters. The results obtained follow the same trend as those presented in the previous section.

Fig. 6 presents the throughput results for an increasing number of primary channels with the time required for spectrum sensing and handoff maintained fixed. As expected, the theoretical maximum throughput can be achieved if the SU has the ability of precognition, whereas the second-best performance is achieved in the case where traffic prediction is employed by the SU.

Fig. 5 illustrates that throughput increases for all four cases as we increase the number of primary channels. This is due to the fact that an increase in the total number of primary channels will lead to an increase in the available transmission opportunities irrespective of whether traffic prediction is used or not. Fig. 5 also demonstrates that with the aid of traffic prediction, we can obtain performance gains as a result of our scheme selecting the primary channels offering the best transmission opportunities.

Fig. 6 shows the impact of the variation in the time required to carry out spectrum sensing and handoff on the average throughput in each time slot. Fig. 6 illustrates that the difference in performance gains between the two spectrum sensing cases with and without traffic prediction augments as we increase the time consumed for spectrum sensing and handoff. This is a result of the reduction in the effective transmission rate in a time slot due to the increment in the time consumed for spectrum sensing and handoff. Since traffic prediction can aid the SU in capturing a transmission opportunity as quickly as possible, this could extend the SU's effective transmission time,

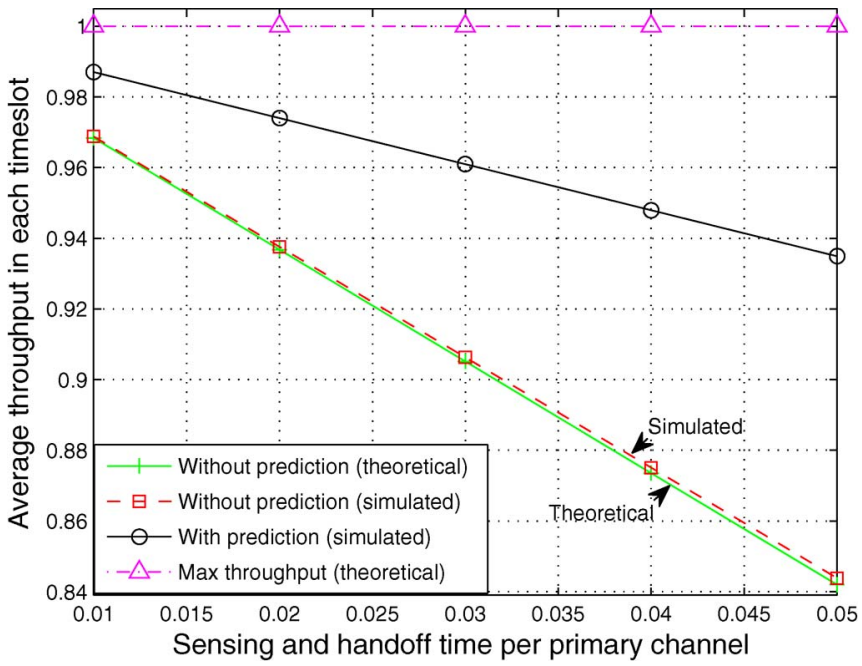

Fig. 6. Average throughput in each time slot for an increasing sensing and handoff time (under a fixed number of primary channels; $N=20$ ).

thus improving the spectrum efficiency. This is particularly true when the time consumed for spectrum sensing and handoff is high, which results in a substantial decrease in throughput for the case where traffic prediction is not incorporated into the spectrum-sensing and access cycle.

\section{CONCLUSION}

In this paper, we have proposed an intelligent selective opportunistic spectrum-sensing and access cycle to enable CR users to make efficient use of the wireless spectrum, which consists of discontinuous frequency bands. The SU determines the optimum sensing order based on its knowledge of the traffic pattern of a primary channel and its prediction of the channel state at a future point in time. The selective spectrum sensing and access cycle is compatible with the QoS requirements of an SU from the point of view of considering the spectrum sensing efficiency in conjunction with transmission capacity when the SU is in the process of establishing the optimum spectrum-sensing order. In this paper, we also presented a theoretical model to evaluate system performance in terms of PLR and throughput and determined the system performance upper bound that may be used as a performance measure for CR networks employing traffic prediction. Finally, our simulation results are in accordance with the numerical results obtained from theory. They also prove that the proposed SOSA based on traffic prediction can improve system efficiency and guarantee the QoS of an SU operating in a discontinuous spectrum environment.

\section{APPENDIX}

In Section V, we need to construct a random process made up of three random variables $K, M, L$ following binomial distribution. We define the random variable in this random process as $V_{K, M, L}$. Because these three are distinct with each other and are convolution characteristics of the moment-generating function, we can obtain the moment-generating function of the random variable $V_{K, M, L}$ as

$$
M_{V}(t)=M_{K}(t) M_{-M}(t) M_{L}(t) .
$$




$$
\begin{aligned}
V_{K, M, L}^{3}= & \sum_{k=0}^{N-S}\left(\begin{array}{c}
N \\
k
\end{array}\right) P^{k}(1-P)^{N-k} \\
& \times\left\{1-\sum_{r=0}^{S-k}\left\{P_{e}^{k-1} \frac{P_{e}}{1-P_{e}} \cdot\left(1-P_{e}\right)^{N-k-1} \pi c s c[\pi(r+k-N)]\right.\right. \\
& \quad\left\{\begin{array}{l}
-\frac{\left(1-P_{e}\right)^{2} \Gamma(1-r)_{2} F_{1}\left(-k, 1-r, 2-k+N-r,\left(1-P_{e}\right)^{2} / P_{e}^{2}\right)}{\Gamma(k-N) \Gamma(2-k+N-r)} \\
\left.\left.\left.\quad+\frac{\left.P_{e}^{2}\left[\frac{\left(1-P_{e}\right)^{2}}{P_{e}^{2}}\right]^{k-M+r} \Gamma(-1-N+r)_{2} F_{1}\left[k-N,-1-N+r, k-N+r,\left(1-P_{e}\right)^{2} / P_{e}^{2}\right)\right]}{\Gamma(-k)}\right\}\right\}\right\}
\end{array}\right.
\end{aligned}
$$

We know that the moment-generating function of binomial distribution is $M_{X}(t)=p e^{t}+q$, where $X$ is a random variable following binomial distribution $B(N, p)$ and the linear translation characteristic of the moment-generating function. Therefore, the foregoing formula can be rewritten as

$M_{V}(t)=\left(P e^{t}+1-P\right)^{N}\left(P_{e} e^{-t}+1-P_{e}\right)^{K}\left(P_{e} e^{t}+1-P_{e}\right)^{N-K}$

where $P$ is the average probability of being idle of a primary channel when we assume that all the primary channels have the same traffic model and traffic characteristic parameters, and $P_{e}$ is the average probability of incorrect prediction of SU. Generally, to obtain the pdf, we need to implement inverse transformation on $M_{V}(t)$ to get an expression in terms of $k$. Obviously, it is not easy and intuitive to get the final solution. However, we could get a conditional expression according to the predefined multiple cases in Section V.

1) $K \leq N-S, M=K, L \geq S$ : For this case, we can separately consider the three random variables because there is no need to compare certain combination of $K$, $M$, and $L$ with the sensing threshold $S$. Therefore, the probability of occurrence of this situation is

$$
\begin{aligned}
V_{K, M, L}^{1}=(1-P)^{N} \sum_{k=0}^{N-S}\left(\begin{array}{c}
N \\
k
\end{array}\right)\left(\frac{P P_{e}}{1-P}\right)^{k} \\
\cdot\left[1-\sum_{l=0}^{S}\left(\begin{array}{c}
N-k \\
l
\end{array}\right) P_{e}^{l}\left(1-P_{e}\right)^{N-K-l}\right] .
\end{aligned}
$$

2) $K \leq N-S, M=K, L<S$ : This case has a complementary relationship with the foregoing case, and therefore, the probability of occurrence of this situation is

$$
\begin{aligned}
V_{K, M, L}^{2}=(1-P)^{N} & \sum_{k=0}^{N-S}\left(\begin{array}{c}
N \\
k
\end{array}\right)\left(\frac{P P_{e}}{1-P}\right)^{k} \\
\cdot & {\left[\sum_{l=0}^{S}\left(\begin{array}{c}
N-k \\
l
\end{array}\right) P_{e}^{l}\left(1-P_{e}\right)^{N-K-l}\right] . }
\end{aligned}
$$

3) $K \leq N-S, M<K, K-M+L>S$ : In this case, we need to consider the joint distribution of the three random variables because we have to compare the magnitude of $K-M+L$ and $S$. To simplify the inverse transformation procedure, we will research the magnitude relationship between $K-M+L$ and $S$ under a certain $K$, which definitely satisfy $0 \leq K \leq N-S$. Therefore, (27) will be

$$
M_{V}(t)=\left[P_{e}+\left(1-P_{e}\right) e^{t}\right]^{K}\left(P_{e} e^{t}+1-P_{e}\right)^{N-K} .
$$

By searching from [17], we find that the probability density of $V_{K, M, L}^{3}$ can be written as (25), shown at the top of the page. ${ }_{2} F_{1}$ in (25) is the regularized hypergeometric function.

\section{REFERENCES}

[1] Spectrum policy task force, ET Docket No. 02-135, Tech. Rep., Fed. Commun. Comm., Nov. 2002.

[2] J. Mitola and G. Q. Maguire, "Cognitive radio: Making software radios more personal," IEEE Pers. Commun., vol. 6, no. 4, pp. 13-18, Aug. 1999.

[3] I. F. Akyildiz, W. Y. Lee, M. C. Vuran, and S. Mohanty, "Next generation/dynamic spectrum access/cognitive radio wireless networks: A survey," Comput. Netw. J., vol. 50, no. 13, pp. 2127-2159, Sep. 2006.

[4] S. Haykin, "Cognitive radio: Brain-empowered wireless communications," IEEE J. Sel. Areas Commun., vol. 23, no. 2, pp. 201-220, Feb. 2005.

[5] M. Gandetto and C. Regazzoni, "Spectrum sensing: A distributed approach for cognitive terminals," IEEE J. Sel. Areas Commun., vol. 25, no. 3, pp. 546-557, Apr. 2007.

[6] S. Mishra, A. Sahai, and R. Brodersen, "Cooperative sensing among cognitive radios," in Proc. IEEE ICC, 2006, pp. 1658-1663.

[7] A. Ghasemi and E. S. Sousa, "Fundamental limits of spectrum-sharing in fading environments," IEEE Trans. Wireless Commun., vol. 6, no. 2, pp. 649-658, Feb. 2007.

[8] L. C. Wang and A. Chen, "On the performance of spectrum handoff for link maintenance in cognitive radio," in Proc. Int. Symp. Wireless Pervasive Comput., May 2008, pp. 670-674.

[9] X. Zhu, L. Shen, and T.-S.P. Yum, "Analysis of cognitive radio spectrum access with optimum channel reservation," IEEE Commun. Lett., vol. 11, no. 4, pp. 304-306, Apr. 2007.

[10] Q. Shi, D. Taubenheim, S. Kyperountas, P. Gorday, and N. Correal, "Link maintenance protocol for cognitive radio system with OFDM PHY," in Proc. IEEE Int. Symp. New Frontiers Dyn. Spectrum Access Netw., Apr. 2007, pp. 440-443.

[11] W. Y. Lee and I. F. Akyildiz, "Optimum spectrum sensing framework for cognitive radio networks," IEEE Trans. Wireless Commun., vol. 7, no. 10, pp. 3845-3857, Oct. 2008.

[12] Y. Chen, Q. Zhao, and A. Swami, "Joint design and separation principle for opportunistic spectrum access in the presence of sensing errors," IEEE Trans. Inf. Theory, vol. 54, no. 5, pp. 2053-2071, May 2008.

[13] M. Hoyhtya, S. Pollin, and A. Mammela, "Performance improvement with predictive channel selection for cognitive radios," in Proc. Cognitive Radio Adv. Spectrum Manag., Feb. 2008, pp. 1-5. 
[14] Z. Wen, T. Luo, W. Xiang, S. Majhi, and Y. Ma, "Autoregressive spectrum hole prediction model for cognitive radio systems," in Proc. IEEE Int. Conf. Commun., 2008, pp. 154-157.

[15] L. Yang, L. Cao, and H. Zheng, "Proactive channel access in dynamic spectrum networks," Phys. Commun., vol. 1, no. 2, pp. 103-111, Jun. 2008.

[16] T. Clancy and B. Walker, "Predictive dynamic spectrum access," in Proc. SDR Forum Tech. Conf., Nov. 2006. [Online]. Available: http://www.cs.umd.edu/ clancy/docs/sdr06-predict.pdf

[17] A. Jeffrey and D. Zwillinger, Table of Integrals, Series and Products, 7th ed. New York: Academic, 2007.

[18] J. Polson, Cognitive Radio Applications in Software Defined Radio. [Online]. Available: http://www.sdrforum.org

[19] G. X. Yuan, R. C. Grammenos, Y. Yang, and W. B. Wang, "Selective spectrum sensing and access based on traffic prediction," in Proc. IEEE PIMRC, 2009.

[20] N. Devroye, P. Mitran, and V. Tarokh, "Achievable rates in cognitive radio channels," IEEE Trans. Inf. Theory, vol. 52, no. 5, pp. 1813-1827, May 2006

[21] A. Goldsmith, S. A. Jafar, I. Maric, and S. Srinivasa, "Breaking spectrum gridlock with cognitive radios: An information theoretic perspective," Proc. IEEE, vol. 97, no. 5, pp. 894-914, May 2009.

[22] R. Etkin, A. Parekh, and D. Tse, "Spectrum sharing for unlicensed bands," IEEE J. Sel. Areas Commun., vol. 25, no. 3, pp. 517-528, Apr. 2007.

[23] G. Ganesan and Y. Li, "Cooperative spectrum sensing in cognitive radio, Part I: Two user networks," IEEE Trans. Wireless Commun., vol. 6, no. 6, pp. 2204-2213, Jun. 2007.

[24] D. I. Kim, L. B. Le, and E. Hossain, "Joint rate and power allocation for cognitive radios in dynamic spectrum access environment," IEEE Trans. Wireless Commun., vol. 7, no. 12, pp. 5517-5527, Dec. 2008.

[25] L. B. Le and E. Hossain, "Resource allocation for spectrum underlay in cognitive radio networks," IEEE Trans. Wireless Commun., vol. 7, no. 12, pp. 5306-5315, Dec. 2008 .

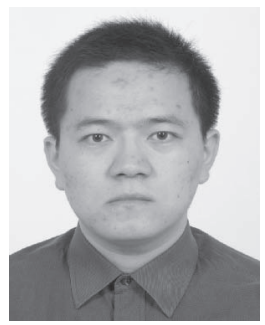

Guangxiang Yuan received the B.S. degree from Huazhong University of Science and Technology University, Wuhan, China, in 2004. He is currently working toward the Ph.D. degree with the Wireless Signal Processing and Networks Laboratory, School of Telecommunications Engineering, Beijing University of Posts and Telecommunications, Beijing, China.

From September 2008 to December 2009, he was a visiting Ph.D. student with the Department of Electronic and Electrical Engineering, University College London (UCL), London, U.K., and an Intern with the Research Institute of British Telecommunication (Adastral Park, Ipswich, U.K.) for three months during the period of researching with UCL. His current research interests include key technologies in beyond-third-generation mobile communication systems, cooperative communication, and intelligent processing in wireless networks.

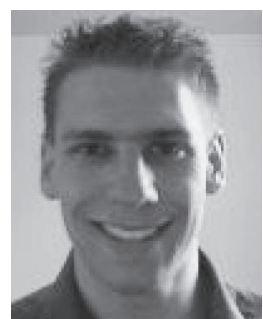

Ryan C. Grammenos received the B.Eng. (Hons.) degree in electronic engineering from Cardiff University, Cardiff, U.K., in 2007 and the M.Eng. degree in electrical and electronic engineering and automatic control from the University of Nice-Sophia Antipolis, Nice, France, in 2008. He is currently working toward the D.Eng. degree with the Telecommunications Research Group, University College London, London, U.K.

His research interests focus on improving the spectrum efficiency in next-generation mobile networks using cognitive radio and orthogonal frequency division multiplexing modulation techniques.

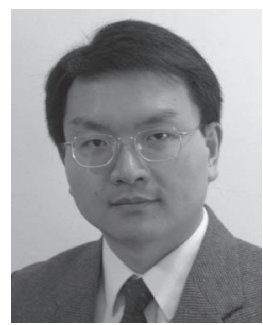

Yang Yang (S'99-M'02) received the B.Eng. and M.Eng. degrees in radio engineering from Southeast University, Nanjing, China, in 1996 and 1999, respectively, and the $\mathrm{Ph} . \mathrm{D}$. degree in information engineering from The Chinese University of Hong Kong, Hong Kong, in 2002

From August 2002 to August 2003, he was an Assistant Professor with the Department of Information Engineering, Chinese University of Hong Kong. From September 2003 to February 2005, he was a Lecturer with the Department of Electronic and Computer Engineering, Brunel University, Uxbridge, U.K. He is currently a Senior Lecturer with the Department of Electronic and Electrical Engineering, University College London, London, U.K., and an Assistant Director with the Shanghai Research Center for Wireless Communications, Chinese Academy of Sciences. He has published over 70 referred journal and conference papers, covering a wide range of research areas such as next-generation mobile communications, wireless ad hoc, sensor and mesh networks, cross-layer algorithm design and performance evaluation, cooperative communications, cognitive radio, dynamic radio resource management, and medium-access control protocols.

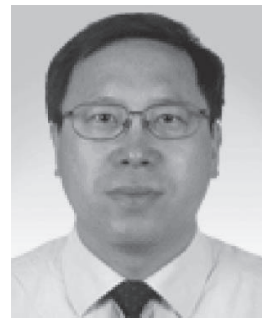

Wenbo Wang (M'94) received the B.S. degree in communication engineering and the M.S. and Ph.D. degrees in signal and information processing from the Beijing University of Posts and Telecommunications (BUPT), Beijing, China, in 1986, 1989, and 1992, respectively.

From October 1992 to 1993, he was a Researcher with ICON Communication Inc., Dallas, TX. He has been a Chair Professor with the School of Information and Communications Engineering, BUPT, since 2000, where he is currently the Executive Dean of the Graduate School. He is the Assistant Director of the Universal Wireless Communication Lab. He has been the Director of the Beijing Institute of Communication since 2002 and an Assistant Director of the National Defense Communication Committee, China Institute of Communication. He has published more than 200 papers and six books and is the holder of 12 patents. His research interests include transmission technology, third-generation and beyond third-generation broadband wireless access, wireless network theory, digital signal processing, orthogonal frequency division multiplexing/code division multiple access, mutiple-input-multiple output, cooperative and cognitive communications, and software radio technology. 